\title{
Multicenter phase II study of antimicrobial prophylaxis in low-risk patients undergoing distal gastrectomy for gastric cancer
}

\author{
Hiroshi Imamura ${ }^{1}$, Hiroshi Furukawa ${ }^{1}$, Shohei Iijima ${ }^{2}$, Seichi Sugihara ${ }^{3}$, Toshimasa Tsujinaka ${ }^{4}$, \\ Hideaki Tsukuma ${ }^{5}$, and Toshio Shimokawa ${ }^{6}$ \\ ${ }^{1}$ Department of Surgery, Sakai City Hospital, 1-1-1 Minamiyasui-cho, Sakai, Osaka 590-0064, Japan \\ ${ }^{2}$ Department of Surgery, Mino Municipal Hospital, Osaka, Japan \\ ${ }^{3}$ Department of Surgery, Hoshigaoka Welfare Annuity Hospital, Osaka, Japan \\ ${ }^{4}$ Department of Surgery, National Hospital Organization Osaka National Hospital, Osaka, Japan \\ ${ }^{5}$ Department of Epidemiology, Osaka Medical Center for Cancer and Cardiovascular Diseases, Osaka, Japan \\ ${ }^{6}$ Department of Ecosocial System, Yamanashi University, Yamanashi, Japan
}

\begin{abstract}
Background. In Japan, antimicrobial prophylaxis (AMP) is typically administered for 3 to 4 days postoperatively for clean-contaminated operations such as distal gastrectomy. This far exceeds the recommended $24 \mathrm{~h}$ or less laid out by the Centers for Disease Control (CDC) guidelines for the prevention of surgical-site infections (SSIs), after a cleancontaminated operation.

Methods. Patients with gastric cancer, which was curable by distal gastrectomy with D2 lymphandectomy, were assigned to a multicenter phase II study. One gram of cefazolin within $30 \mathrm{~min}$ of the first surgical incision and intraoperative supplements of $1 \mathrm{~g}$ of cefazolin every $3 \mathrm{~h}$ were administered. AMP was not administered after skin closure unless postoperative infection occurred. The occurrence of SSIs was monitored until postoperative day 30 as the primary endpoint of this study.

Results. In 56 patients, three SSIs (5.4\%) and one remote site infection (1.8\%) developed. No other postoperative infections were observed.

Conclusion. Our data indicate that the CDC guidelines regarding AMP may also be sufficient for patients undergoing clean-contaminated operations in Japan.
\end{abstract}

Key words Antimicrobial prophylaxis - Surgical-site infection - Gastric cancer - CDC guidelines - Postoperative infection

\section{Introduction}

According to the Centers for Disease Control (CDC) guidelines for the prevention of surgical-site infections

Offprint requests to: $\mathrm{H}$. Imamura Received: August 22, 2005 / Accepted: November 24, 2005 Presented at the 40th annual meeting of the American Society of Clinical Oncology, New Orleans, LA, June 5-8, 2004.
(SSIs) [1], a first-generation cephem or penicillin, as antimicrobial prophylaxis (AMP), should be administered for clean or clean-contaminated operations. Administration of AMP within $30 \mathrm{~min}$ of the first surgical incision, with intraoperative supplemental administration every 3 or $4 \mathrm{~h}$ and postoperative administration for $24 \mathrm{~h}$ or less are the recommended timings and durations for the administration of AMP.

According to the results of a questionnaire completed by 3823 surgeons in Japan in 2002, 56\% of surgeons reported that they administered AMP for cleancontaminated operations until the third or fourth postoperative day, while only $7 \%$ of them reported that they administered AMP for clean-contaminated operations for 2 days or less. The reason that doctors in Japan have been reluctant to adopt the CDC guidelines is that they are afraid of an increased incidence of SSIs. Recently, however, many doctors have come to recognize the importance of the preoperative administration and intraoperative supplemental administration of AMP. According to guidelines developed by the Japanese Society of Chemotherapy (JSC) for the prevention of SSI, 3 to 4 days after the operation is an appropriate duration for the administration of AMP for cleancontaminated operations. The justification given for this is that, in Japan, a wider area of lymphadenectomy is performed for malignant tumors of the upper gastrointestinal tract when compared with those performed in the United States and European countries.

In order to clarify the discrepancy regarding the appropriate duration of AMP administration between the guidelines of the CDC and those of the JSC, we decided to conduct a multicenter phase II clinical study of AMP in low-risk patients undergoing distal gastrectomy for gastric cancer. This operation for malignant tumors of the upper gastrointestinal tract is classified as a cleancontaminated operation. 


\section{Methods}

\section{Study design}

We conducted a multicenter phase II study that was approved by the institutional review boards of all participating hospitals and conducted in observance of the Declaration of Helsinki, as well as good clinical practice guidelines. The primary focus of this study was to measure the incidence of SSI. Secondary considerations included monitoring the incidence of remote site infections, pyrexia in excess of $38^{\circ} \mathrm{C}$, and other complications.

\section{Eligibility}

After completion of the informed consent process, patients were included in the study if they met the following eligibility criteria: histologically proven gastric cancer which was curable by distal gastrectomy with D2 lymphadenectomy and which was classified as a cleancontaminated operation at preoperative diagnosis; and an American Society of Anesthesiologists (ASA) score of 1 or 2 . None of the following conditions were permitted: active or uncontrolled infection; neoadjuvant chemotherapy; or the occurrence of secondary malignancy.

\section{Treatment}

Patient history was reviewed, a full-body examination was performed, and eligibility-related criteria were recorded. Preoperatively, patients were provisionally registered with the central data center by facsimile to confirm that the eligibility requirements were met; that there were no conditions that would bar inclusion in the study; and that valid informed consent had been obtained. After confirmation of eligibility by the central data center, the following schedule of treatment was administered.

In patients for whom hair removal was necessary at and around the incision site, scissors or hair clippers were used instead of a razor. Use of a razor was avoided because it was feared stubble from shaving could possibly produce minute abrasions, which, in turn, would increase the likelihood of infection [2]. One gram of cefazolin, a first-generation cephem, was administered as AMP within $30 \mathrm{~min}$ of the first surgical incision to all patients undergoing scheduled distal gastrectomy with D2 lymphadenectomy. Furthermore, intraoperative supplements of $1 \mathrm{~g}$ of cefazolin were administered every $3 \mathrm{~h}$. No AMP was administered after skin closure unless postoperative infection occurred. This treatment schedule was not used for patients with an intraoperative change in operative method or for patients undergoing contaminated operations due to some intraoperative trouble, these patients were excluded from evaluation.

\section{Evaluation}

Operative time, amount of bleeding, degree of lymphadenectomy, reconstructive method, anastomotic method, and presence of drainage tubes were evaluated as intraoperative factors. SSIs, pyrexia in excess of $38^{\circ} \mathrm{C}$, remote site infections, and any other complications were evaluated as postoperative factors. These conditions were monitored for 30 days after the operation and the data were sent to the central data center by facsimile within 50 days of the operation. The degrees of lymphadenectomy were in accordance with the second English edition of the Japanese Classification of gastric carcinoma [3]. SSIs were checked by an infection control doctor (ICD) belonging to the infection control team (ICT) at each institution, using the National Nosocomial Infection Surveillance (NNIS) system of the CDC [4], during the patients' stay in the hospital. Also, after leaving hospital, all patients were instructed to consult their doctors if they noticed any discharge from the surgical site. Moreover, even if there were no symptoms indicative of an SSI after leaving the hospital, surgical sites for all patients were checked by each doctor during follow-up examinations.

\section{Statistics}

This study was designed to test the null hypothesis that the true incidence of SSI probability is more than the clinically insignificant level of $17 \%$. If the hypothesis is rejected, we will accept the specified alternative hypothesis that the true incidence of SSI probability is at least a target level of $6.7 \%$. Therefore, the sample size was 50 patients, with alpha, 0.05 , and beta, 0.8 . We determined the target number of patients in this phase II study to be 55.

\section{Results}

\section{Patient characteristics}

A total of 65 patients with gastric cancer were provisionally enrolled in this study, between August 2002 and June 2003, at five medical facilities in Osaka, Japan. Nine patients had to be excluded from evaluation (because of a change in the operative method to a total gastrectomy $[n=7]$; change of classification to a contaminated operation $[n=1]$; and a violation of the protocol regarding the treatment schedule as judged retrospectively by the external review committee $[n=$ 1]). This left 56 patients who were eligible for inclusion in this study. 
Table 1. Preoperative demographic characteristics

\begin{tabular}{lc} 
Characteristic & Values are means \pm SD or no. $(\%)$ \\
\hline Male & $38(67.9)$ \\
Female & $18(32.1)$ \\
Median age, years & $64 \pm 10$ \\
ASA score & \\
$\quad 1$ & $44(78.6)$ \\
2 & $12(21.4)$
\end{tabular}

ASA, American Society of Anesthesiologists

Table 2. Intraoperative demographic characteristics

\begin{tabular}{lc}
\hline Characteristic & Values are means \pm SD or no. (\%) \\
\hline Operative time, min & $186 \pm 49$ \\
Intraoperative & \\
$\quad$ supplemental & \\
$\quad$ administration & \\
$\quad$ of AMP & $33(58.9)$ \\
No & $23(41.1)$ \\
Yes & $301 \pm 189$ \\
Blood loss during operation, ml & \\
D2 lymphandectomy & $56(100)$ \\
Yes & $0(0)$ \\
No & $46(82.1)$ \\
Reconstructive methods & $10(17.9)$ \\
Billroth-I & \\
Roux-en-Y & $19(33.9)$ \\
Anastomotic methods & $37(66.1)$ \\
Handsewn & \\
Autosuture & $56(100)$ \\
Presence of drainage tube & $0(0)$ \\
Yes &
\end{tabular}

Pretreatment demographic characteristics of the 56 study patients are listed in Table 1 . Table 2 lists the intraoperative characteristics of the 56 study patients. The mean operative time was $186 \pm 49 \mathrm{~min}$ (range, 89 to $354 \mathrm{~min})$. Of the total of 56 study patients, 33 patients $(58.9 \%)$ received $1 \mathrm{~g}$ of cefazolin as AMP only once at $30 \mathrm{~min}$ before surgical incision, because total operative time did not exceed $3 \mathrm{~h}$, while 23 patients $(41.1 \%)$ received $1 \mathrm{~g}$ of cefazolin as AMP once at $30 \mathrm{~min}$ before surgical incision and a second intraoperative supplemental administration of $1 \mathrm{~g}$ of cefazolin $3 \mathrm{~h}$ after the first incision.

\section{Infections and other complications after operations}

Table 3 shows data regarding infections and other complications after operation. In the 56 study patients, three SSIs were detected, these consisted of two organ/space SSIs and one deep incisional SSI, as determined using the NNIS system of the CDC. All three of the SSIs were checked during the hospital stay, and no SSI occurred after patients left the hospital. Therefore, the total inci-
Table 3. Infections and other complications after operation

\begin{tabular}{lc} 
Characteristics & No. $(\%)$ \\
\hline SSI & \\
Not detected & $53(94.6)$ \\
Detected & $3(5.4)$ \\
$\quad$ Organ/space SSI & $2(3.6)$ \\
$\quad$ Deep incisional SSI & $1(1.8)$ \\
$\quad$ Superficial incisional SSI & $0(0.0)$ \\
Pyrexia more than $38^{\circ} \mathrm{C}$ & \\
Not detected & $38(67.9)$ \\
Detected & $18(32.1)$ \\
Remote site infection & $55(98.2)$ \\
Not detected & $1(1.8)$ \\
Detected & $56(100)$ \\
Other complications & $0(0.0)$ \\
$\quad$ Not detected & \\
Detected & \\
SSI, surgical site infection &
\end{tabular}

dence of SSIs, which, as stated earlier was the main focus of this study, was observed to be $5.4 \%$ (3/56). Through a bacteriological analysis, it was determined that Escherichia coli accounted for one organ/space SSI; the other two infections were not cultured, however. All three SSIs were successfully treated during the course of the study. A remote site infection in the urinary tract developed in 1 patient $(1.8 \%)$.

\section{Discussion}

According to a questionnaire survey in Japan [5], regarding patients undergoing clean-contaminated operations, only $2 \%$ of surgeons administered AMP just during operation. This survey also reported that most surgeons supported AMP administration until 3 to 7 days after a clean-contaminated operation, and $38 \%$ of surgeons administered AMP until 7 days after the operation. These results suggested that surgeons believed that long-term administration of antibiotics could keep the incidence of SSIs low. Recent medical opinion regarding the prophylaxis of SSI, however, appears to favor the use of pre- and/or intraoperative AMP only for the majority of cases, with remedial antibiotics used only in patients in whom postoperative infection occurs. According to the CDC guidelines for the prevention of SSIs [1], the surgical wound is originally classified into four categories: clean, clean-contaminated, contaminated, and dirty-infected. It is stated that AMP should be administered for clean and clean-contaminated operations, while remedial antibiotics should be administered only for contaminated and dirty-infected operations.

The administration of cefazolin, a first-generation cephem agent, is recommended as AMP for operations on the upper gastrointestinal tract in which aerobe 
gram-positive coccus is frequently detected [6,7]. Moreover, Higgins et al. [8] point out that there is a marked cost difference among the different antibiotics and that cefazolin is less expensive than many other antibiotics. Accordingly, the administration of cefazolin as AMP is recommended for operations on the upper gastrointestinal tract.

It is believed that the timing of the first administration of AMP should be within $30 \mathrm{~min}$ of the first surgical incision, in order to gain the optimum concentration $[9,10]$. Furthermore, intraoperative supplemental administration of AMP is necessary every 3 or 4 hours after the first incision until skin closure to maintain optimum concentration in the blood and tissue [11].

As described above, it is relatively clear what kind of antibiotics should be administered and when the antibiotics should be started for clean-contaminated operations such as distal gastrectomy for gastric cancer. There is no discrepancy between the guidelines of the CDC and those of the JSC regarding this matter. However, there is a difference of opinion regarding the duration of administration of AMP for these operations between the guidelines of the CDC and those of the JSC. Of course, the duration of the administration of AMP is assumed to be as short as possible, because a longer duration of administration tends to induce bacteria with resistance to antibiotics and also tends to be unjustifiably expensive [12]. The results of some prospective randomized studies suggest that AMP is not needed for low-risk patients undergoing elective laparoscopic cholecystectomy, which is classified as a cleancontaminated operation, as is distal gastrectomy $[8,13,14]$. However, it still remains to be definitively proven that a shorter duration of administration of AMP does not in any way increase the incidence of SSIs.

In a previously unpublished study that we conducted, the incidence of SSI was $6.7 \%(2 / 30)$ in a patient group similar to that in the present study, the only difference being that the duration of AMP administration for this group was until the third postoperative day. By the end of their course of treatment they had received $1 \mathrm{~g}$ of cefazolin eight to nine times; once or twice during the operation; once on the night of the operative day; and twice a day for 3 days after the operation. By contrast, the patients in the present study received $1 \mathrm{~g}$ of cefazolin as AMP only once or twice during their course of treatment. Therefore, our data, showing that the incidence of SSI was $5.4 \%$ in the present study, are assumed to demonstrate that the protocol of this study is feasible for low-risk patients undergoing distal gastrectomy for gastric cancer.

A phase III study is now ongoing, conducted by our study group (Osaka Gastrointestinal Chemotherapy Study Group; OGSG) to confirm that the administra- tion of AMP after skin closure may be unnecessary for distal gastrectomy for gastric cancer in Japan.

\section{Conclusion}

This study shows that administration of AMP within $30 \mathrm{~min}$ of the first surgical incision and supplemental administration of AMP every $3 \mathrm{~h}$ before skin closure may be enough to keep the incidence of SSI low. A phase III study is necessary to confirm that administration of AMP after skin closure may be unnecessary for clean-contaminated operations such as distal gastrectomy for gastric cancer.

Acknowledgments This work was supported in part by a Grant-in-Aid (17S-3) from the Ministry of Health, Labor, and Welfare of Japan.

\section{References}

1. Mangram AJ, Horan TC, Pearson ML, Silver LC, Jarvis WR. Guidelines for prevention of surgical site infection, 1999. Hospital Infection Control Practices Advisory Committee. Infect Control Hosp Epidemiol 1999;20:231-2.

2. Alexander JW, Fischer JE, Boyajian M, Palmquist J, Morris MJ. The influence of hair-removal methods on wound infections. Arch Surg 1983;118:347-52.

3. Japanese Gastric Cancer Association. Classification of gastric carcinoma. 2nd English ed. Gastric Cancer 1998;1:10-24.

4. National Nosocomial Infection Surveillance System. Atlanta: U.S. Department of Health and Human Services, Public Health Service, Centers for Disease Control and Prevention (CDC); 1999.

5. Shinagawa N, Mashita K, Iwai S, Yokoyama T, Takeyama H. A questionnaire survey on the theory of postoperative infection prophylaxis. Jpn J Gastroenterol Surg 2000;33:1559-63.

6. Nichols RL. Antibiotic prophylaxis in surgery. J Chemother 1989;1:170-8.

7. Bumpous JM, Johnson JT. The infected wound and its management. Otolaryngol Clin North Am 1995;28:987-1001.

8. Higgins A, London J, Charland S, Ratzer E, Clark J, Haun W, et al. Prophylactic antibiotics for elective laparoscopic cholecystectomy: are they necessary? Arch Surg 1999;134:611-4.

9. Page CP, Bohnen JM, Fletcher JR, McManus AT, Solomkin JS, Wittmann DH. Antimicrobial prophylaxis for surgical wounds. Guidelines for clinical care. Arch Surg 1993;128:79-88.

10. Classen DC, Evans RS, Pestotnik SL, Horn SD, Menlove RL, Burke JP. The timing of prophylactic administration of antibiotics and risk of surgical-wound infection. N Engl J Med 1992;326:2816.

11. Ohge H, Takesue Y, Yokoyama T, Murakami Y, Hiyama E, Yokoyama Y, et al. An additional dose of cefazolin for intraoperative prophylaxis. Surg Today 1999;29:1233-6.

12. Harbarth S, Samore MH, Lichtenberg D, Carmeli Y. Prolonged antibiotic prophylaxis after cardiovascular surgery and its effect on surgical site infections and antimicrobial resistance. Circulation 2000;101:2916-21.

13. Dobay KJ, Freier DT, Albear P. The absent role of prophylactic antibiotics in low-risk patients undergoing laparoscopic cholecystectomy. Am Surg 1999;65:226-8.

14. Tocchi A, Lepre L, Costa G, Liotta G, Mazzoni G, Maggiolini F. The need for antibiotic prophylaxis in elective laparoscopic cholecystectomy. Arch Surg 2000;135:67-70. 